\title{
A Common Fixed Point Theorem for Multivalued Mappings Through $T$-weak Commutativity
}

\author{
I. Kubiaczyk and Bhavana Deshpande
}

\begin{abstract}
In this paper, we prove a common fixed point theorem for a singlevalued and the multivalued mappings by using $T$-weak commutativity condition. We also show that continuity of any mapping is not needed for the existence of the common fixed point.
\end{abstract}

\section{InTRODUCTION}

In 1976, Jungck [5] proved a common fixed point theorem for commuting maps, generalizing the Banach's fixed point theorem. Sessa [20] generalized the notion of commutativity and defined weak commutativity. Further, Jungck [6] introduced more generalized commutativity, so called compatibility and generalized some results of Singh and Singh [23] and Fisher [3]. Kaneco [9] extended the concept of weakly commuting mappings for multivalued set up and extended result of Jungck [5]. Kaneko and Sessa [10] extended the concept of compatible mappings for multivalued mappings and generalized the result of Kubiak [12]. In 1998, Jungck and Rhoades [7] extended weak compatibility in the settings of singlevalued and multivalued mappings. Pant $[16,17,18,19]$ initiated the study of non compatible mappings and introduced $R$-weak commutativity of mappings. He also showed that for single-valued mappings pointwise $R$-weak commutativity is equivalent to weak compatibility at the coincidence points. Shahzad and Kamran [21] and Singh and Mishra [22] have independently extended the idea of $R$-weak commutativity to the settings of single and multivalued mappings.

In [22], Singh and Mishra introduced the notion of (IT)-commutativity for a hybrid pair of single-valued and multivalued mappings and showed that a pointwise $R$-weakly commuting hybrid pair need not be weakly compatible. However at the coincidence points pointwise $R$-weak commutativity for hybrid pairs is equivalent to (IT)-commutativity.

Recently, Kamaran [8] introduced the notion of $T$-weak commutativity for a single-valued and a multivalued mapping and showed that it is weaker condition than (IT)-commutativity and weak compatibility of hybrid pair.

2000 Mathematics Subject Classification. Primary: 47H10, 54H25.

Key words and phrases. Common fixed point, $T$-weakly commuting mappings. 
In their paper, Kaneko [9] and Kaneko and Sessa [10] have assumed a pair of single-valued and multivalued mapping which are continuous at $\mathrm{X}$ and could prove the existence of a coincidence point. For the existence of a common fixed point an additional hypothesis is needed. They have also remarked whether or not the continuity of two mappings is really needed in the proof.

Kubiaczyk and Mustafa Ali [11], Krzyska and Kubiaczyk [14] and many others have proved common fixed point theorems for multivalued mappings.

Asad and Ahmad [1] extended the results of Fisher [2] for multivalued mappings using condition of weak commutativity or compatibility and proved that existence of common fixed point can be achieved by the continuity of the single-valued mapping only, the continuity of the multivalued mappings are not needed.

In this paper, we improve results of Asad and Ahmad [1] by taking $T$-weak commutativity in place of weak commutativity or in place of compatibility, without assuming continuity of any mapping.

\section{PReliminaries}

Let $(X, d)$ be a metric space and suppose that $C B(X)$ denotes the set of nonempty closed and bounded subsets of $X$.

For $A, B$ in $C B(X)$ we denote

$$
\begin{aligned}
D(A, B) & =\inf \{d(a, b): a \in A, b \in B\} \\
D(x, A) & =\inf \{d(x, a): a \in A\} \\
H(A, B) & =\max \{\sup \{D(a, B): a \in A\}, \sup \{D(A, b): b \in B\}\} .
\end{aligned}
$$

Kuratowski [13] showed that $(C B(X), H)$ is a metric space with the distance function $H$, moreover $(C B(X), H)$ is complete in the event that $(X, d)$ is complete.

Lemma 1 ([15]). Let $A, B \in C B(X)$, then for $\varepsilon>0$ and $a \in A$ there exists $b \in B$ such that $d(a, b) \leq H(A, B)+\varepsilon$. If $A$ and $B$ are compact then one can find $b \in B$ such that $d(a, b) \leq H(A, B)$.

Definition 1 ([9]). Let $(X, d)$ be a metric space, $F: X \rightarrow C B(X)$ and $T: X \rightarrow$ $X$. Then the pair $\{F, T\}$ is said to be weakly commuting if for each $x \in X$, $T F(x) \in C B(X)$ and $H(F T x, T F x) \leq D(T x, F x)$.

Definition $2([10])$. Let $(X, d)$ be a metric space, $F: X \rightarrow C B(X)$ and $T: X \rightarrow$ $X$. Then the pair $\{F, T\}$ is said to be compatible if and only if $T F x \in C B(X)$ for each $x \in X$ and $H\left(F T x_{n}, T F x_{n}\right) \rightarrow 0$ whenever $\left\{x_{n}\right\}$ is a sequence in $X$ such that $F x_{n} \rightarrow M \in C B(X)$ and $T x_{n} \rightarrow t \in M$.

Definition 3 ([7]). The mapping $T: X \rightarrow X$ and $F: X \rightarrow C B(X)$ are weakly compatible if they commute at their coincidence points that is if $F T u=T F u$ whenever $T u \in F u$.

Definition $4([4,22])$. The mapping $T: X \rightarrow X$ and $F: X \rightarrow C B(X)$ are said to be (IT)-commuting at $x \in X$ if $T F x \subseteq F T x$. 
Definition 5 ([21]). The mappings $T: X \rightarrow X$ and $F: X \rightarrow C B(X)$ are said to be $R$-weakly commuting if, for given $x \in X, T F x \in C B(X)$ and there exists some positive real number $R$ such that $H(T F x, F T x) \leq R d(T x, F x)$.

Definition $6([8])$. Let $T: X \rightarrow X$ and $F: X \rightarrow C B(X)$ the hybrid pair $\{T, F\}$ is said to be $T$-weakly commuting at $x \in X$ if $T T x \in F T x$.

Example 1. Let $X=[0, \infty)$ with the usual metric. Define $T: X \rightarrow X$ and $F: X \rightarrow C B(X)$ by $T x=3 x$ and $F x=[0,3+3 x]$ for all $x \in X$. Then for all $x \in X, T x \in F x, T T x=9 x \in[0,3+9 x]=F T x$. Therefore the pair $\{T, F\}$ is $T$ weakly commuting but not (IT)-commuting because $T F x=[0,9+9 x] \nsubseteq F T x=$ $[0,3+9 x]$. Also note that $T$ and $F$ are not weakly compatible. Moreover, if $\left\{x_{n}\right\}$ is a sequence in $X$ such that $x_{n} \rightarrow 1$. Then $\lim _{n \rightarrow \infty} T x_{n}=3 \in[0,6]=\lim _{n \rightarrow \infty} F x_{n}$ and $\lim _{n \rightarrow \infty} H\left(T F x_{n}, F T x_{n}\right)=6$. Therefore the mappings $F$ and $T$ are not compatible.

Remark 1 ([8]). $\quad$ (i) Let $T: X \rightarrow X$ and $F: X \rightarrow C B(X)$. The hybrid pair $\{T, F\}$ is (IT)-commuting at the coincidence points implies that it is $T$-weakly commuting but $T$-weakly commuting hybrid pair is neither (IT)-commuting nor weakly compatible in general.

(ii) If $F$ is single-valued mapping then $T$-weak commutativity at the coincidence points is equivalent to the weak compatibility.

(iii) It is known [17] that pointwise $R$-weak commutativity is minimal condition for the existence of fixed point.

\section{Main Result}

Theorem 1. Let $(X, d)$ be a complete metric space, $T: X \rightarrow X$ and $F, G: X \rightarrow$ $C B(X)$, satisfying

(3.1) $F(X) \cup G(X) \subseteq T(X)$;

(3.2) the pairs $\{F, T\}$ and $\{G, T\}$ are $T$-weakly commuting at their coincidence points;

$H(F x, G y) \leq \alpha \frac{[D(F x, T y)]^{2}+[D(G y, T x)]^{2}}{D(F x, T y)+D(G y, T x)}+\beta d(T x, T y)$

$x \neq y, F x \neq F y, G x \neq$ Gy for all $x, y \in X, \alpha, \beta \geq 0,2 \alpha+\beta<1$, whenever $D(F x, T y)+D(G y, T x) \neq 0$ and $H(F x, G y)=0$, whenever $D(F x, T y)+$ $D(G y, T x)=0$.

Then there exists a point $z$ in $X$ such that $z=T z \in F z \cap G z$.

Proof. Assume $\theta=\frac{\alpha+\beta}{1-\alpha}$. Let $x_{0} \in X$ and $y_{1}$ be an arbitrary point in $F x_{0}$. Choose $x_{1} \in X$ such that $y_{1}=T x_{1}$. This is possible as $F(X) \subseteq T(X)$. By Lemma 1, we can find $y_{2} \in G x_{1}$ such that

$$
d\left(y_{1}, y_{2}\right) \leq H\left(F x_{0}, G x_{1}\right)+\frac{1-\alpha}{1+\alpha} \theta .
$$


Choose $x_{2} \in X$ such that $y_{2}=T x_{2}$. This is also possible as $G(X) \subseteq T(X)$. Also we can find $y_{3} \in F x_{2}$ such that

$$
d\left(y_{2}, y_{3}\right) \leq H\left(F x_{2}, G x_{1}\right)+\frac{1-\alpha}{1+\alpha} \theta^{2} .
$$

Inductively, having selected $y_{2 n}=T x_{2 n} \in G x_{2 n-1}$, choose $y_{2 n+1}=T x_{2 n+1} \in$ $F x_{2 n}$ such that

$$
d\left(y_{2 n+1}, y_{2 n}\right) \leq H\left(F x_{2 n}, G x_{2 n-1}\right)+\frac{1-\alpha}{1+\alpha} \theta^{2 n} .
$$

Then having selected $y_{2 n+1}$, choose $y_{2 n+2}=T x_{2 n+2} \in G x_{2 n+1}$ such that

$$
d\left(y_{2 n+1}, y_{2 n+2}\right) \leq H\left(F x_{2 n}, G x_{2 n-1}\right)+\frac{1-\alpha}{1+\alpha} \theta^{2 n+1} .
$$

Since conditions (3.1) and (3.3) are similar to that of Asad and Ahmad [1]. So as proved in [1], we can prove that $\left\{y_{n}\right\}=\left\{T x_{n}\right\}$ is a Cauchy sequence in $X$. Since $X$ is complete, there exists a point $z$ in $X$ such that $y_{n} \rightarrow z$ as $n \rightarrow \infty$. Since $F(X) \subseteq T(X)$, there exists a point $p \in X$ such that $T p=z$. By (3.3), we have

$$
\begin{aligned}
D(T p, F p) \leq D(T p, & \left.G x_{2 n-1}\right)+H\left(G x_{2 n-1}, F p\right) \\
D(T p, F p) \leq D(T p, & \left.G x_{2 n-1}\right)+ \\
& +\alpha \frac{\left[D\left(F p T x_{2 n-1}\right)\right]^{2}+\left[D\left(G x_{2 n-1}, T p\right)\right]^{2}}{D\left(F p, T x_{2 n-1}\right)+D\left(G x_{2 n-1}, T p\right)}+\beta d\left(T p, T x_{2 n-1}\right), \\
D(T p, F p) \leq D(T p, & \left.G x_{2 n-1}\right)+ \\
& +\alpha\left[D\left(F p, T x_{2 n-1}\right)+D\left(G x_{2 n-1}, T p\right)\right]+\beta d\left(T p, T x_{2 n-1}\right) .
\end{aligned}
$$

On letting $n \rightarrow \infty$, we get

$$
D(z, F p) \leq \alpha \cdot D(F p, z),
$$

a contradiction. Therefore $z \in F p$ that is $z=T p \in F p$. So $p \in X$ is a coincidence point of $F$ and $T$.

Similarly since $G(X) \subseteq T(X)$, there exists a point $q \in X$ such that $T q=z$. By (3.3), we have

$$
\begin{aligned}
& D(T q, G q) \leq D\left(T q, F x_{2 n}\right)+H\left(F x_{2 n}, G q\right), \\
& D(T q, G q) \leq D\left(T q, F x_{2 n}\right)+\alpha \frac{\left[D\left(F x_{2 n}, T q\right)\right]^{2}+\left[D\left(G q, T x_{2 n}\right)\right]^{2}}{D\left(F x_{2 n}, T q\right)+D\left(G q, T x_{2 n}\right)}+\beta d\left(T x_{2 n}, T q\right), \\
& D(T q, G q) \leq D\left(T q, F x_{2 n}\right)+\alpha\left[D\left(F x_{2 n}, T q\right)+D\left(G q, T x_{2 n}\right)\right]+\beta d\left(T x_{2 n}, T q\right) .
\end{aligned}
$$

On letting $n \rightarrow \infty$, we get

$$
D(z, G q) \leq \alpha \cdot D(z, G q),
$$

a contradiction. Therefore $z \in G q$ that is $T q \in G q$. So $q \in X$ is a coincidence point of $G$ and $T$. 
Since the hybrid pair $\{T, F\}$ is $T$-weakly commuting at coincidence point $p \in X$. Thus $T T p \in F T p$ that is $T z \in F z$. Similarly $T$-weakly commutativity of $\{T, G\}$ at coincidence point $q \in X$ gives $T z \in G z$. By (3.3), we have

$$
\begin{aligned}
& d\left(T x_{2 n}, T z\right) \leq H\left(G x_{2 n-1}, F z\right), \\
& d\left(T x_{2 n}, T z\right) \leq \alpha \frac{\left[D\left(F z T x_{2 n-1}\right)\right]^{2}+\left[D\left(G x_{2 n-1}, T z\right)\right]^{2}}{D\left(F z, T x_{2 n-1}\right)+D\left(G x_{2 n-1}, T z\right)}+\beta d\left(T z, T x_{2 n-1}\right), \\
& d\left(T x_{2 n}, T z\right) \leq \alpha\left[D\left(F z, T x_{2 n-1}\right)+D\left(G x_{2 n-1}, T z\right)\right]+\beta d\left(T z, T x_{2 n-1}\right) .
\end{aligned}
$$

On letting $n \rightarrow \infty$, we get

$$
d(z, T z) \leq(2 \alpha+\beta) d(z, T z),
$$

a contradiction giving there by $z=T z$. Thus we have shown that $z=T z \in$ $F z \cap G z$. This completes the proof.

Remark 2. (i) For $\alpha=0$, we get an extension of the well known Banach fixed point theorem.

(ii) For $\beta=0$, we get a new result.

Remark 3. Kaneko [9] and Kaneko and Sessa [10] assumed the continuity of both single valued and multivalued mappings. They have questioned however whether the continuity of both the mappings is really needed in the proof. In our Theorem 1 , we have shown that existence of a common fixed point can be achieved without assuming continuity of any mapping.

Remark 4. We improve Theorem 1 and Theorem 3.2 of Asad and Ahmad [1] by relaxing weak commutativity to $T$-weak commutativity and compatibility to $T$-weak commutativity respectively. We also remove condition of continuity of any mapping.

Remark 5 ([1]). The condition in the hypothesis " $x \neq y, F x \neq F y, G x \neq G y$ " is necessary since the Theorem 1 fails for $F$ and $G$ taken as constant mappings.

\section{REFERENCES}

[1] A.J. Asad and Z. Ahmad, Common fixed point of multivalued mappings with weak commutativity conditions, Radovi, Math. Vol. 9(1999), 119-124.

[2] B. Fisher, Mappings satisfying rational inequality, Nanta Math., 12(1979), 195-199.

[3] B. Fisher, Common fixed point of four mappings, Bull. Inst. Math. Acad. Sinica, 11(1983), $103-113$.

[4] S. Ithoh and W. Takahashi, Single-valued mappings, multivalued mappings and fixed point theorems, J. Math. Anal. Appl., 59(1977), 514-521.

[5] G. Jungck, Commutating mappings and fixed points, Amer. Math. Monthly, 83(1976), 261263.

[6] G. Jungck, Compatible mappings and common fixed points, Internat J. Math. Math. Sci., 9(4) (1986), 771-779.

[7] G. Jungck and B. E. Rhoades, Fixed point for set valued functions without continuity, Ind. J. Pure and Appl. Math., 29(3) (1998), 227-238. 
[8] T. Kamran, Coincidence and fixed points for hybrid strict contractions, J. Math. Anal. Appl., 299 (2004), 235-241.

[9] H. Kaneko, A common fixed point of weakly commuting multivalued mappings, Math. Japon., 33(5) (1988), 741-744.

[10] H. Kaneko and S. Sessa, Fixed point theorems for compatible multivalued and single-valued mappings, Internat. J. Math. Math. Sci., 12(2) (1989), 257-262.

[11] I. Kubiaczyk and N. Mostafa Ali, A multivalued fixed point theorems in non Archimedean vector spaces, Novi Sad J. Math., 26(2) (1996), 111-115.

[12] T. Kubiak, Fixed point theorems for contractive type multivalued mappings, Math. Japon., 30(1985), 89-101.

[13] K. Kuratowski, Topology, Vol. 1, Academic press, 1966.

[14] S. Kyzyska and I. Kubiaczyk, Fixed point theorems for upper semicontinuous and weakly upper semicontinuous multivalued mappings, Math. Japonica, 47, No. 2(1998), 237-240.

[15] S.B. Nadler, Multivalued contractions mappings, Pacific J. Math. 30(2) (1969), 475-488.

[16] R.P. Pant, Common fixed points of non-commuting mappings, J. Math. Anal. Appl., 188 (1994), 436-440.

[17] R.P. Pant, Common fixed point theorems for contractive maps, J. Math. Anal. Appl. 236 (1998), 251-258.

[18] R.P. Pant, Common fixed points of Lipschitz type mapping pairs, J. Math. Anal. Appl., 240 (1999), 280-283.

[19] R.P. Pant, Discontinuity and fixed points, J. Math. Anal. Appl., 240(1999), 284-289.

[20] S. Sessa, On weak commutativity condition of mappings in fixed point consideration, Publ. Inst. Math., 32(46) (1982), 149-155.

[21] N. Shahzad and T. Kamran, Coincidence points and R-weakly commuting maps, Arch. Math. (Brno), 37(2001), 179-183.

[22] S. L. Singh and S. N. Mishra, Coincidence and fixed points of non self hybrid contraction, J. Math. Anal. Appl., 256(2001), 486-497.

[23] S. L. Singh and S. P. Singh, A fixed point theorem, Ind. J. Pure Appl. Math., 11(1980), $1584-1586$.

Faculty of Mathematics and Computer Science Adem Mickiewicz University

Ul. Umultowska 87

61-614 POZNAN

POLAND

E-mail address: kuba@amu.edu.pl

Department of Mathematics

Govt. Arts and Science P. G. College

RATLAM-457001 (M.P.)

INDIA

E-mail address: bhavnadeshpande@yahoo.com

Current address:

"Sukhakarta"

90-Rajiv Nagar (Near Kasturba Nagar)

Ratlam-457001 (M. P.)

India

E-mail address: bhavnadeshpande@yahoo.com 